\title{
Transcriptomics of atopy and atopic asthma in white blood cells from children and adolescents
}

\author{
Yale Jiang ${ }^{1,2,9}$, Olena Gruzieva ${ }^{3,9}$, Ting Wang ${ }^{1}$, Erick Forno (1) ${ }^{1}$, Nadia Boutaoui ${ }^{1}$, \\ Tao Sun ${ }^{4}$, Simon K. Merid ${ }^{3}$, Edna Acosta-Pérez ${ }^{5}$, Inger Kull ${ }^{3}$, Glorisa Canino ${ }^{5}$, \\ Josep M. Antó ${ }^{6}$, Jean Bousquet ${ }^{7,8}$, Erik Melén $\mathbb{\circledR}^{3,10}$, Wei Chen $\mathbb{\oplus}^{1,10}$ and \\ Juan C. Celedón ${ }^{1,10}$ \\ Affiliations: ${ }^{1}$ Division of Pulmonary Medicine, Dept of Pediatrics, UPMC Children's Hospital of Pittsburgh, \\ University of Pittsburgh, Pittsburgh, PA, USA. ${ }^{2}$ School of Medicine, Tsinghua University, Beijing, China. \\ ${ }^{3}$ Institute of Environmental Medicine, Karolinska Institutet, Stockholm, Sweden. ${ }^{4}$ Dept of Biostatistics, \\ Graduate School of Public Health, University of Pittsburgh, Pittsburgh, PA, USA. ${ }^{5}$ Behavioral Sciences \\ Research Institute, University of Puerto Rico, San Juan, Puerto Rico. ' ISGlobal, Barcelona Institute for Global \\ Health, Barcelona, Spain. ${ }^{7}$ CESP, Inserm U1018, Villejuif, France. ${ }^{8}$ University Hospital, Montpellier, France. \\ ${ }^{9}$ These two authors contributed equally to this work. ${ }^{10}$ These three authors are joint senior authors.
}

Correspondence: Juan C. Celedón, Division of Pediatric Pulmonary Medicine, UPMC Children's Hospital of Pittsburgh, 4401 Penn Avenue, Pittsburgh, PA 15224, USA. E-mail: juan.celedonachp.edu

@ERSpublications

In Puerto Rican and Swedish children, a TWAS identified 59 DEGs by atopy and 40 DEGs by atopic asthma, including SIGLEC8 and IL17RB. Transcriptomic data from WBCs could be used to develop predictive models of atopy/atopic asthma in prospective studies. http://ow.ly/kF6v30nYnYC

Cite this article as: Jiang Y, Gruzieva O, Wang T, et al. Transcriptomics of atopy and atopic asthma in white blood cells from children and adolescents. Eur Respir J 2019; 53: 1900102 [https://doi.org/10.1183/ 13993003.00102-2019].

ABSTRACT Early allergic sensitisation (atopy) is the first step in the development of allergic diseases such as atopic asthma later in life. Genes and pathways associated with atopy and atopic asthma in children and adolescents have not been well characterised.

A transcriptome-wide association study (TWAS) of atopy and atopic asthma in white blood cells (WBCs) or whole blood was conducted in a cohort of 460 Puerto Ricans aged 9-20 years (EVA-PR study) and in a cohort of 250 Swedish adolescents (BAMSE study). Pathway enrichment and network analyses were conducted to further assess top findings, and classification models of atopy and atopic asthma were built using expression levels for the top differentially expressed genes (DEGs).

In a meta-analysis of the study cohorts, both previously implicated genes (e.g. IL5RA and IL1RL1) and genes not previously reported in TWASs (novel) were significantly associated with atopy and/or atopic asthma. Top novel genes for atopy included SIGLEC8 $\left(\mathrm{p}=8.07 \times 10^{-13}\right)$, SLC29A1 $\left(\mathrm{p}=7.07 \times 10^{-12}\right)$ and SMPD3 $\left(\mathrm{p}=1.48 \times 10^{-11}\right)$. Expression quantitative trait locus analyses identified multiple asthma-relevant genotype-expression pairs, such as rs2255888/ALOX15. Pathway enrichment analysis uncovered 16 significantly enriched pathways at adjusted $\mathrm{p}<0.01$, including those relevant to T-helper cell type 1 (Th1) and Th2 immune responses. Classification models built using the top DEGs and a few demographic/parental history variables accurately differentiated subjects with atopic asthma from nonatopic control subjects (area under the curve 0.84).

We have identified genes and pathways for atopy and atopic asthma in children and adolescents, using transcriptome-wide data from WBCs and whole blood samples. 


\section{Introduction}

Over the last few decades, the prevalence of allergic diseases has increased in the USA and worldwide. Sensitisation to allergens (atopy) in early life precedes the development of allergic diseases later in childhood, including food allergies, allergic rhinitis and atopic asthma [1]. Puerto Rican and Swedish children bear a high burden of atopy and asthma [1-3]. In studies of school-aged children, estimates of the prevalence of asthma and allergic rhinitis in Puerto Ricans were $16.1 \%$ and $48.4 \%$, respectively $[2,4]$. Among Swedish children aged 7-8 years, estimates of the prevalence of current wheeze and allergic rhinitis were $13 \%$ and $13.8 \%$, respectively [5].

Recent meta-analyses of genome-wide association studies (GWASs) identified 18 susceptibility loci for asthma [6] and 41 susceptibility loci for allergic rhinitis [7]. However, such loci accounted for a small proportion of the heritability of asthma $(\sim 3.5 \%)$ and allergic rhinitis $(\sim 7.8 \%)$, and alternative approaches such as transcriptomics could help identify the "missing heritability" of atopic diseases. Yet another complementary approach is to study intermediate phenotypes such as atopy, as susceptibility genes for atopy often overlap with those for common allergic diseases [7].

In contrast to the many GWASs conducted to date, there have been few transcriptome-wide association studies (TWASs) of atopy and asthma, all limited by modest sample size and no or insufficient replication [8-12]. For example, a study of targeted RNA sequencing of 105 genes in nasal brushings from subjects with $(n=50)$ and without $(n=50)$ asthma showed that nasal transcriptomic profiles could identify subjects with IL13-driven asthma and T-helper cell type 2 (Th2)-skewed immune responses [9]. In another study (including mostly adults), RNA sequencing profiles from nasal brushings were used to first develop a panel of 90 genes to differentiate subjects with mild-to-moderate asthma $(n=53)$ from control subjects $(\mathrm{n}=97)$, and then test the "classifier panel" in 40 subjects with $(\mathrm{n}=13)$ and without $(\mathrm{n}=27)$ asthma [10]. Although the panel performed well in training cohorts, external replication was limited and thus no individual genes could confidently be identified as conferring susceptibility to asthma.

While airway epithelial studies have shown promising results, blood samples are easy to collect and often available in epidemiological studies. A genome-wide analysis of transcriptomics in circulating CD19 ${ }^{+}$ B-lymphocytes from 41 adults provided suggestive evidence of increased IL4R expression in subjects with dust mite allergy and asthma compared with control subjects [11]. In another study in young adolescents with asthma, microarray expression profiles from $\mathrm{CD}^{+}$T-lymphocytes were differentially expressed by atopic status [12] and five "atopic signature" genes were then nominally replicated in a cohort of 30 subjects. A later integrative study in asthma controls focused on the transcriptomic components in blood that vary with degree of asthma control [13].

Here, we report the findings from the first TWAS of atopy and atopic asthma in white blood cells (WBCs) from children and adolescents in Puerto Rico, with validation studies in a cohort of Swedish children.

\section{Methods}

Further details are given in the supplementary material.

\section{Study population}

The EVA-PR (Epigenetic Variation and Childhood Asthma in Puerto Ricans) study is a case-control study of asthma in Puerto Ricans aged 9-20 years, recruited using an approach similar to that used in previous studies in the metropolitan area of San Juan (Puerto Rico) [14, 15]. Of the 543 participating children, 460 had measurements of IgE to five common allergens in Puerto Rico (dust mite (Der p 1), cockroach (Bla g 2), cat dander (Fel d 1), dog dander (Can f 1) and mouse urinary protein (Mus m 1)) [16], WBC count and differential, and RNA from WBCs, and were thus included in the current analysis. The study was approved by the institutional review boards of the University of Puerto Rico (San Juan) and the University of Pittsburgh (Pittsburgh, PA, USA). Written parental consent and assent were obtained from all participants.

\section{RNA sequencing and data pre-processing}

Sequencing was performed using $350 \mathrm{ng}$ of high-quality RNA extracted from WBCs after removing haemoglobin (RNA integrity number $>7$ ). Library preparation was done using the TruSeq Stranded Total RNA Library Prep Kit with the Ribo-Zero Gold High Throughput Kit (Illumina, San Diego, CA, USA), according to the manufacturer's protocol. Libraries were run on the NextSeq 500 using the NextSeq 500/550 High Output Kit version 2 (Illumina).

Quality control for raw RNA sequencing FASTQ files was performed using FastQC [17]. Low-quality reads and 3' adapters were trimmed with Trim Galore! [18] and Cutadapt [19]. Trimmed reads were then aligned to reference human genome hg19 with STAR [20] and subsequently annotated in the Illumina 
iGenomes database using RSEM [21]. Samples with low alignment percentage and genes with low expression were removed from downstream analyses. After pre-processing, a total of 16880 genes and 460 samples were retained in the final analysis.

Principal components were calculated from genome-wide genotypic data, which were obtained using the HumanOmni2.5 BeadChip platform (Illumina), as previously described [22]. Imputation of nongenotyped single nucleotide polymorphisms (SNPs) was performed with the Imputation Server [23], using the Haplotype Reference Consortium r1.1 2016 [24] as the reference panel.

\section{The BAMSE study}

We also analysed whole blood samples using Affymetrix Human Transcriptome Array 2.0 (Affymetrix, Santa Clara, CA, USA) in 269 16-year-old children selected from BAMSE (Children [Barn], Allergy, Milieu, Stockholm, Epidemiology), a Swedish population-based cohort (with oversampling of asthma and rhinitis cases) [25]. After quality control (RNA integrity number >6) and outlier detection, gene expression data from 250 blood samples were included in the current analysis of atopy and atopic asthma with available WBC count and differential. Allergen-specific IgE levels were measured using the ImmunoCAP System (Thermo Fisher/Phadia, Uppsala, Sweden).

\section{Statistical analyses}

In EVA-PR and BAMSE, atopy was defined as at least one positive $\operatorname{IgE}\left(\geqslant 0.35 \mathrm{IU} \cdot \mathrm{mL}^{-1}\right)$ to the allergens tested. Atopic asthma was defined as current asthma (physician-diagnosed asthma and current wheeze) and atopy in EVA-PR, and as current asthma (assessed using a modified version of the GA ${ }^{2}$ LEN questionnaire2) and atopy in BAMSE [26].

Differentially expressed genes (DEGs) were analysed based on the raw count table using DESeq2 [27] for RNA sequencing data in EVA-PR and LIMMA for microarray data in BAMSE. In both EVA-PR and BAMSE, multivariable models of atopic asthma were adjusted for age, sex, batch label and the proportions (percentages) of WBC subtypes (eosinophils, lymphocytes, monocytes and neutrophils). All multivariable models of atopy were additionally adjusted for asthma status. In EVA-PR, all models were additionally adjusted for the first five principal components derived from genotypes, to account for population stratification. A zero-mean normal prior was put on the nonintercept coefficients to ensure that fold changes were independent of the choice of reference level. Next, we conducted a meta-analysis of the cohort-specific results using a fixed effect model with in-house code using the R package meta [28, 29]. The false discovery rate (FDR) approach was applied to adjust for multiple testing.

In EVA-PR, we performed an expression quantitative trait loci (eQTL) analysis (at a distance $<1 \mathrm{Mb}$ from gene start and end sites) for the top findings in the meta-analysis of atopy using the $\mathrm{R}$ package Matrix eQTL [30].

\section{Pathway and network analysis}

Ingenuity Pathway Analysis was performed for the top DEGs (FDR-adjusted $\mathrm{p}<0.1$ ) in the meta-analysis of atopy and atopic asthma to identify enriched canonical pathways and gene networks [31]. We then calculated the partial correlation between gene expression levels in our discovery cohort, first in all subjects and then separately by asthma status, using the R package FastGGM [32], a powerful tool to detect conditional gene networks under the framework of the Gaussian graphical model.

\section{Classification models of atopy and atopic asthma}

Logistic regression with penalised maximum likelihood was applied to build classification models of atopy and atopic asthma in EVA-PR. We first built two models for comparison: one including only demographic and parental history variables (age, sex and parental history of asthma) as predictors, and another including demographic variables, parental history and gene expression levels of randomly chosen genes. Next, we built a model using the top DEGs in our meta-analysis, and another using the top DEGs plus demographic variables and parental history. Models were built separately for atopy and atopic asthma.

\section{Results}

The main characteristics of study participants are shown in table 1. In EVA-PR, subjects with atopy were significantly more likely to be male and to have asthma than control subjects. Compared with participants in EVA-PR, those in BAMSE had, as expected for a population-based cohort study, a lower proportion of asthmatic subjects and a narrower age range. 
TABLE 1 Characteristics of participants in the EVA-PR and BAMSE studies

\begin{tabular}{|c|c|c|c|c|}
\hline & \multicolumn{2}{|c|}{ EVA-PR ${ }^{\#}$} & \multicolumn{2}{|c|}{ BAMSE $^{\text {II }}$} \\
\hline & Atopy & No atopy & Atopy & No atopy \\
\hline Subjects & 300 & 160 & 134 & 116 \\
\hline Age years & $15.2(10-20)$ & $15.7(10-20)$ & $16.7(16.0-17.9)$ & $16.7(15.9-18.1)$ \\
\hline Female & $131(43.7)$ & $87(54.4)$ & $47(35.1)$ & $59(50.9)$ \\
\hline Asthma & $165(55)$ & $63(39.4)$ & 42 (31.3) & $10(8.6)$ \\
\hline \multicolumn{5}{|l|}{ Sensitisation to allergens } \\
\hline Dust mite (Der $p$ 1) & $282(94)$ & & 48 (35.8) & \\
\hline Cat dander (Fel d 1) & 49 (16.3) & & 72 (53.7) & \\
\hline Dog dander (Can f 1) & $168(56)$ & & $85(63.4)$ & \\
\hline Mouse urinary protein (Mus m 1) & $12(4)$ & & Not measured & \\
\hline Cockroach (Bla g 2) & $174(58)$ & & Not measured & \\
\hline
\end{tabular}

Data are presented as $n$, mean (range) or $n(\%) .{ }^{\#}: n=460 ;{ }^{~}$ : $n=250$.

TWAS of atopy

In the multivariable analysis of atopy, which was adjusted for WBC subtype and other covariates, we identified 69 DEGs in EVA-PR at FDR-adjusted $\mathrm{p}<0.05$. In BAMSE, there were no DEGs at FDR-adjusted $\mathrm{p}<0.05$, but 17 of the 69 DEGs in EVA-PR were differentially expressed at $\mathrm{p}<0.05$, with similar estimates of effect size across the two studies (supplementary table S1). Next, to increase the statistical power of DEG analysis, we combined the results from the two cohorts in a transcriptome-wide meta-analysis, which identified 59 DEGs by atopy, at an FDR-adjusted $\mathrm{p}<0.05$ (figure 1a and supplementary figure S1). Table 2 lists the top 20 genes (by p-value) in this meta-analysis, for which the direction of the association between gene expression and atopy was consistent across the two studies.

For the top DEGs by atopy, we found 43 enriched pathways at FDR-adjusted $\mathrm{p}<0.05$. Table 3 lists the top 20 enriched pathways, of which the two most significant ones were the Th2 pathway and the Th1/Th2 activation pathway. Other top pathways included those for nerve growth factor (NGF) and adenine/ adenosine salvage I.

\section{TWAS of atopic asthma}

We then conducted a transcriptomic analysis of atopic asthma, finding substantial overlap between the results from this analysis and those for atopy (figure $1 \mathrm{~b}$ and supplementary table S2). We identified 49
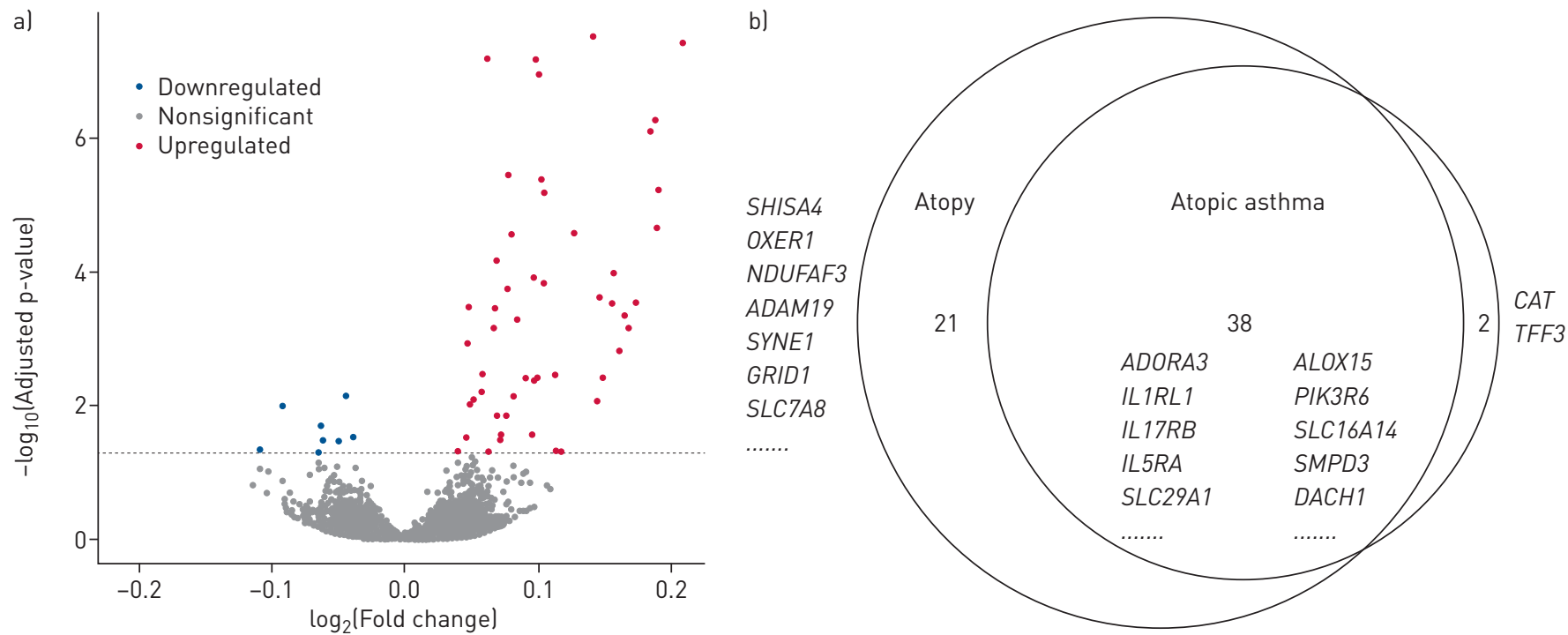

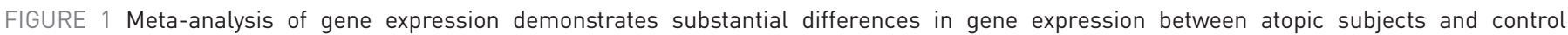

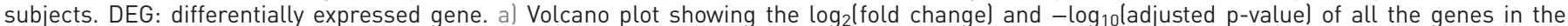

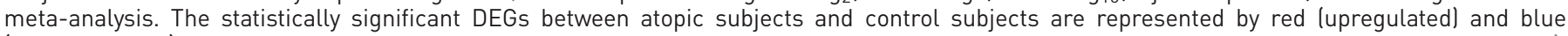
(downregulated) symbols. The statistical criterion for a gene to be considered differentially expressed was false discovery rate-adjusted $p<0.05$. b).

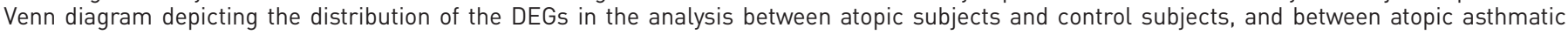
subjects and nonatopic nonasthmatic control subjects. 
TABLE 2 Top differentially expressed genes from the meta-analysis of atopy

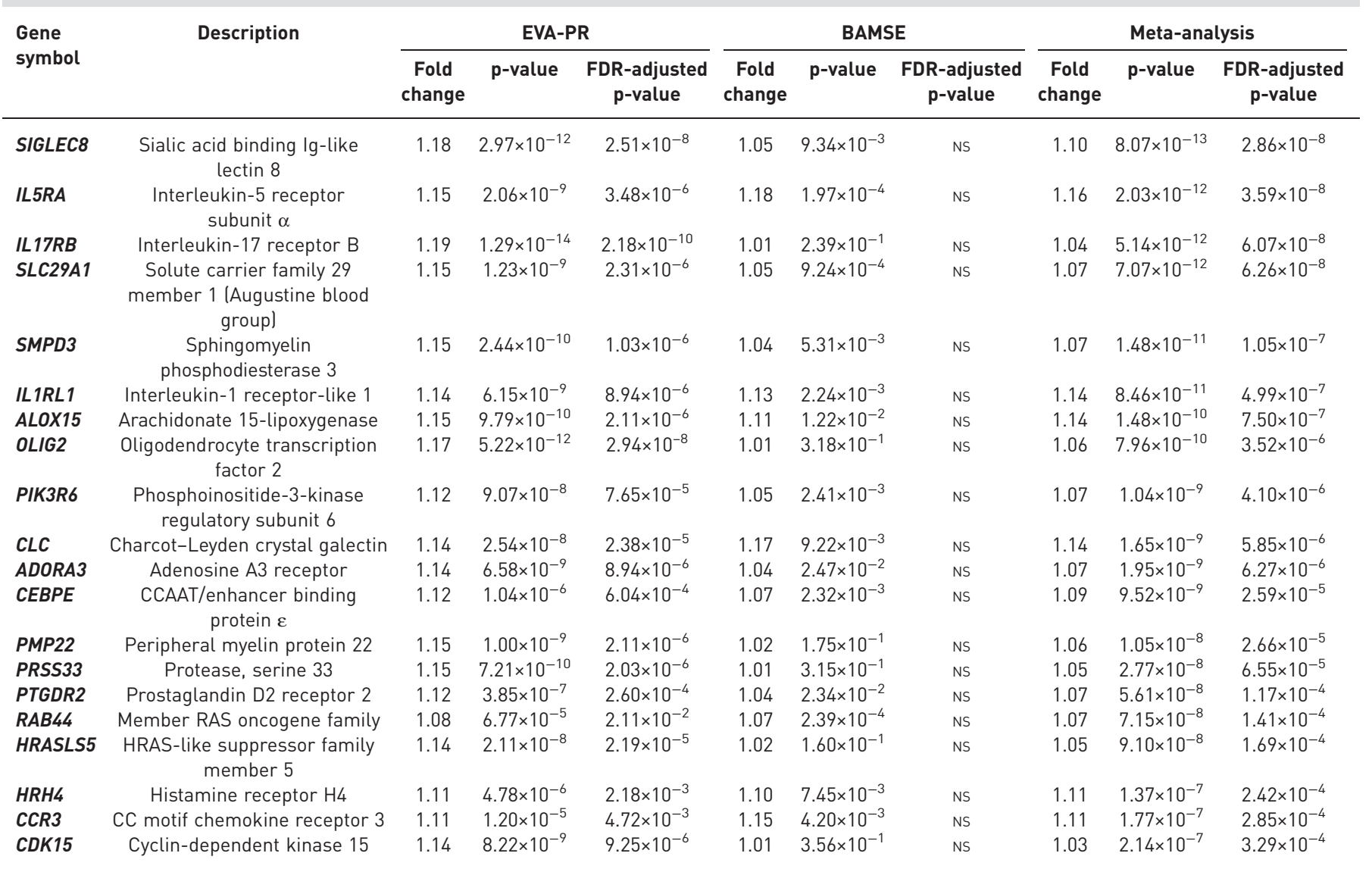

FDR: false discovery rate; Ns: nonsignificant (i.e. FDR-adjusted $p>0.05$ ). All models were adjusted for age, sex, asthma status, batch and proportions of white blood cell subtypes (eosinophils, lymphocytes, neutrophils and monocytes). Models in EVA-PR were additionally adjusted for principal components.

DEGs by atopic asthma in EVA-PR at FDR-adjusted $\mathrm{p}<0.05$. In BAMSE, there were no DEGs at FDR-adjusted $\mathrm{p}<0.05$, but five of the 49 DEGs by atopic asthma in EVA-PR were differentially expressed at $\mathrm{p}<0.05$ in BAMSE, with similar estimates of effect size. 40 DEGs were then identified in the meta-analysis at FDR-adjusted $\mathrm{p}<0.05$. For atopic asthma, a pathway enrichment analysis revealed pathways related to lipid metabolism (e.g. docosahexaenoic acid signalling) and arachidonic acid signalling (e.g. eicosanoids signalling) (supplementary table S3).

\section{eQTL analysis}

Next, we conducted a cis-eQTL analysis in EVA-PR, which identified 3772 SNPs that were significantly associated with our top genes from the expression analysis for atopy at FDR-adjusted $\mathrm{p}<0.05$ (supplementary table S4). Such SNPs included multiple eQTLs in the GTEx database (e.g. rs2255888 in ALOX15 and rs35092096 in CCR3) [33]. Of interest, multiple SNPs were associated with expression of IL1RL1 (a known asthma-susceptibility gene) [6].

\section{Network analysis}

A network analysis was performed to illustrate interactions among our top DEGs by atopy (supplementary figure S2), first in all subjects and then separately in atopic subjects and in control subjects. Partial correlation among these genes remained strong, even after conditioning on global expression profile. This was particularly true for the gene most differentially expressed, SIGLEC8. We also found that some strong correlations in control subjects weakened significantly in atopic children, such as those for the gene pair IL34 and CYSLTR2.

We next performed hierarchical clustering with log-transformed transcripts per kilobase million values in EVA-PR, based on the 59 DEGs (FDR-adjusted $\mathrm{p}<0.05$ ) in the meta-analysis of atopy, identifying four 
TABLE 3 Top functional Ingenuity pathways enriched by the top differentially expressed genes in the meta-analysis of atopy

\begin{tabular}{|c|c|c|}
\hline Ingenuity canonical pathway & $-\log _{10}$ (Adjusted p-value) & Gene symbol \\
\hline Th2 pathway & 3.83 & CCR3, PTGDR2, IL17RB, IL1RL1, PIK3R6 \\
\hline Th1 and Th2 activation pathway & 3.41 & CCR3, PTGDR2, IL17RB, IL1RL1, PIK3R6 \\
\hline Type II diabetes mellitus signalling & 2.75 & CACNA1D, PIK3R6, CACNG8, SMPD3 \\
\hline MSP-RON signalling pathway & 2.74 & CSF1, PIK3R6, RPS6KA2 \\
\hline FcyRIIB signalling in B-lymphocytes & 2.54 & CACNA1D, PIK3R6, CACNG8 \\
\hline $\begin{array}{l}\text { Role of macrophages, fibroblasts and endothelial cells in rheumatoid } \\
\text { arthritis }\end{array}$ & 2.35 & IL1RL1, CSF1, PIK3R6, FZD1, CEBPE \\
\hline Amyotrophic lateral sclerosis signalling & 2.21 & CACNA1D, GRID1, PIK3R6 \\
\hline CREB signalling in neurons & 2.21 & CACNA1D, GRID1, PIK3R6, CACNG8 \\
\hline UVA-induced MAPK signalling & 2.21 & PIK3R6, RPS6KA2, SMPD3 \\
\hline Adenine and adenosine salvage I & 2.18 & $A P R T$ \\
\hline Role of osteoblasts, osteoclasts and chondrocytes in rheumatoid arthritis & 2.11 & ILIRL1, CSF1, PIK3R6, FZD1 \\
\hline nNOS signalling in skeletal muscle cells & 2.08 & CACNA1D, CACNG8 \\
\hline NGF signalling & 2.08 & PIK3R6, RPS6KA2, SMPD3 \\
\hline Atherosclerosis signalling & 2.05 & CCR3, ALOX15, CSF1 \\
\hline Role of tissue factor in cancer & 2.02 & CSF1, PIK3R6, RPS6KA2 \\
\hline Cellular effects of sildenafil (Viagra) & 2.01 & CACNA1D, MYH9, CACNG8 \\
\hline DHA signalling & 1.88 & ALOX15, PIK3R6 \\
\hline$\alpha$-tocopherol degradation & 1.88 & CYP4F12 \\
\hline Tetrapyrrole biosynthesis II & 1.78 & ALAS2 \\
\hline PKC $\theta$ signalling in T-lymphocytes & 1.75 & CACNA1D, PIK3R6, CACNG8 \\
\hline
\end{tabular}

Th: T-helper cell; MSP: macrophage stimulating protein; RON: RON protein tyrosine kinase/receptor d'origine nantais; CREB: cAMP response element binding protein; UVA: ultraviolet A; MAPK: mitogen-activated protein kinase; nNOS: neuronal nitric oxide synthase; NGF: nerve growth factor; DHA: docosahexaenoic acid; PKC: protein kinase C.

individual clusters (figure 2). In this analysis, the prevalence of atopy differs between the first two clusters, but not between the last two clusters (supplementary figure S3). However, when we compared eosinophil proportions in samples across these four clusters, we found that the samples with higher expression of the top DEGs for atopy had more eosinophils in peripheral blood (figure 3). We then tested for an association between expression levels of the top DEGs for atopy or atopic asthma and the measured proportions of each cell subset in peripheral blood (supplementary table S5). Of the top 59 DEGs for atopy, 51 genes were significantly associated (FDR-adjusted $\mathrm{p}<0.05$ ) with the proportion of eosinophils in peripheral blood. However, a few of the top DEGs were associated with the proportions of other cell types (e.g. APRT and TMEM160 with lymphocyte proportions, and PEPD with neutrophil proportions). All top 40 DEGs for atopic asthma were significantly associated with eosinophils in peripheral blood. Next, we deconvolved the expression profile of WBCs based on known cell proportions and permutations in order to investigate cell type-specific differential expression using CIBERSORT [34]. 38 genes were significantly enriched in eosinophils (FDR-adjusted $\mathrm{p}<0.05$ ). Of these 38 genes, 24 were among the top DEGs for atopy and 20 were among the top DEGs for atopic asthma.

\section{Classification models for atopy and atopic asthma}

Finally, we built classification models in our discovery cohort using only demographic variables and parental history, achieving area under the curve (AUC) values of 0.56 for atopy and 0.74 for atopic asthma. These results were similar after adding expression levels for a random set of genes (data not shown). When using top DEGs alone as predictors, the models reached AUC values of 0.77 and 0.81 for atopy and atopic asthma, respectively. However, the performance of the classification models improved substantially after expression levels of the top DEGs, demographic variables and parental history were included as predictors, achieving AUC values of 0.77 for atopy and 0.84 for atopic asthma (figure 4). For atopy, we used 51 genes (FDR-adjusted $\mathrm{p}<0.05$ in the meta-analysis for atopy and available in both cohorts), while for atopic asthma we included the top 33 genes (FDR-adjusted $\mathrm{p}<0.05$ in the meta-analysis for atopic asthma and available in both cohorts).

\section{Discussion}

In a TWAS of WBCs, we identified DEGs by atopy and atopic asthma in 460 Puerto Rican children. In parallel, we conducted a TWAS of atopy and atopic asthma in 250 Swedish children, which yielded nonsignificant results due to the small sample size, but yielded replication of some of our top findings in Puerto Rican children at $\mathrm{p}<0.05$, in the same direction of association and with similar estimates of effect 


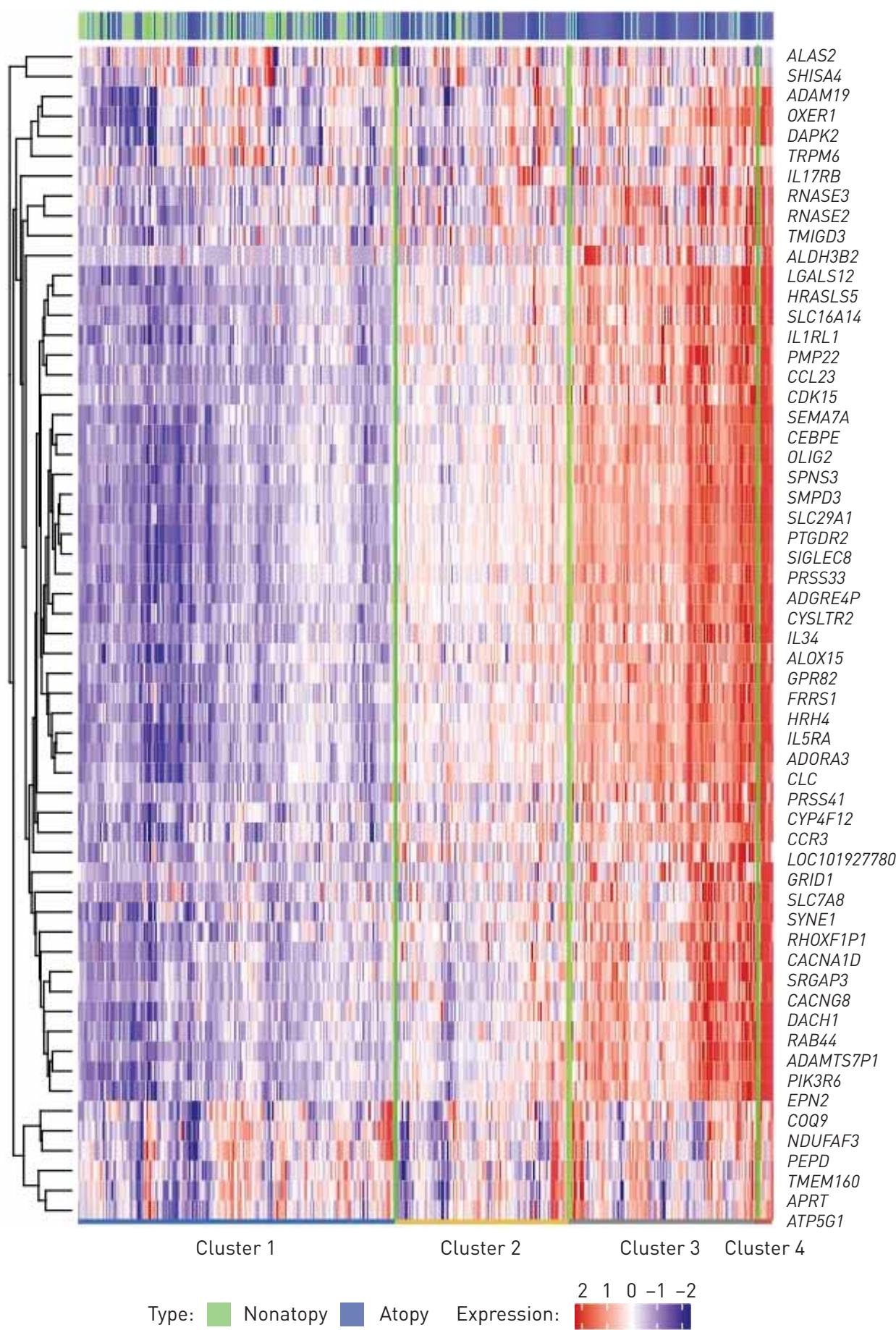

FIGURE 2 Hierarchical clustering based on the top differentially expressed genes in the meta-analysis. Four clusters are identified, with most of the genes upregulated from cluster 1 to cluster 4.

size. Next, we conducted a meta-analysis of TWASs from both study cohorts, identifying 59 DEGs by atopy and 40 DEGs by atopic asthma.

In our meta-analysis, the top DEG by atopy was SIGLEC8, which has an extracellular binding domain that induces apoptosis in eosinophils and inhibits FceRI-dependent mediator release in mast cells [35]. SNPs in SIGLEC8 were associated with asthma and eosinophilic oesophagitis in candidate gene studies [36, 37], but this gene was not associated with asthma in a recent meta-analysis of GWASs [6]. Of interest, expression of one of the top 20 genes in our meta-analysis of atopy (OLIG2, encoding oligodendrocyte transcription factor 2) was previously reported to downregulate RNA and protein levels of SIGLEC8 in 


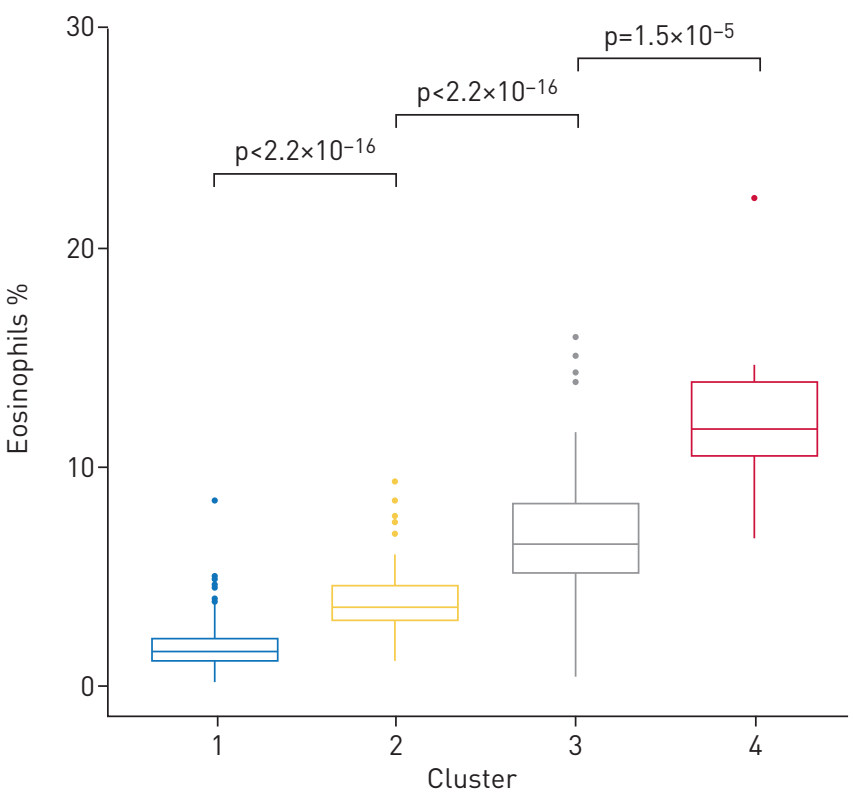

FIGURE 3 Eosinophil proportion comparison for each cluster indentified in figure 2, showing significant difference both globally and between clusters individually in the Kruskal-Wallis test. Box-and-whisker plots show median with interquartile range (IQR) (boxes) and upper/lower limit within 1.5 IQR from the box range (whiskers); outliers are indicated by individual data points.

peripheral blood eosinophils [38]. Moreover, expression levels of OLIG2 and CCL23 (one of our top DEGs for atopic asthma) in whole blood were significantly associated with suboptimal disease control in a meta-analysis of data from 693 ethnically diverse children and adults [13]. Both OLIG2 and CCL23 are TREM-1 (triggering receptor expressed in myeloid cells-1)/lipopolysaccharide signalling genes, and TREM-1 is a key amplifier of inflammatory responses induced by pattern recognition receptors.

Other top study results are supported by experimental evidence or findings from other human studies. SLC29A1 is involved in adenosine-regulated inhibition of IgE-dependent degranulation of mast cells [39]. IL5RA, a gene implicated in asthma in our prior study [40], regulates Th2 cell differentiation and Th2 cell effector functions [41]. Moreover, IL5RA and ALOX15 (also among our top genes) were both significantly associated with eosinophil count in a transcriptomic analysis of blood samples from subjects with asthma [36]. This is consistent with our prior findings for total IgE [42] and, together with the results from our hierarchical cluster analysis and other complementary analyses, further emphasises the key role of eosinophil-dependent mechanisms in the pathogenesis of atopy and atopic asthma.
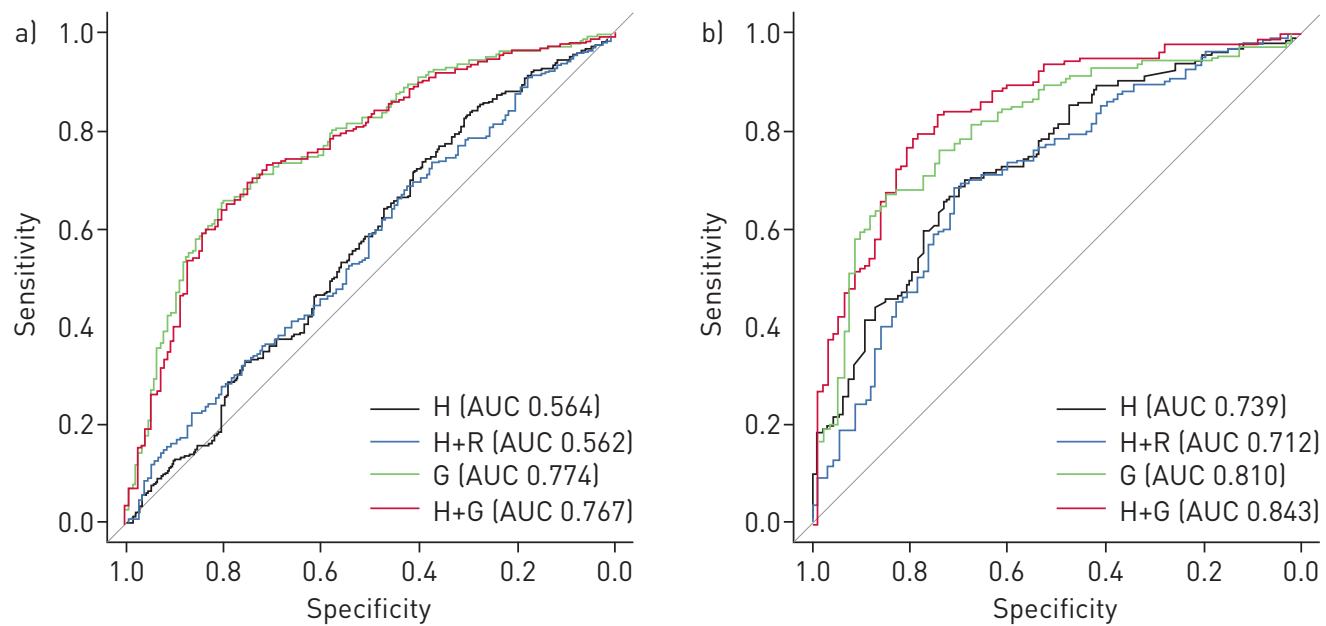

FIGURE 4 Classification models of a) atopy and b) atopic asthma. $\mathrm{H}$ : age, sex and asthma history of parents as predictors; R: expression profile of randomly chosen genes (the same number as G) as predictors; G: expression profile of top differentially expressed gene predictors (false discovery rate-adjusted $p<0.05$ in the meta-analysis); AUC: area under the receiver operating characteristic curve. 
Our findings for $I L 17 R B$ are particularly interesting. In mouse models, allergen challenge triggers the interleukin (IL)-4 pathway, which promotes allergic airway inflammation and airway responsiveness. In turn, activation of IL-4 pathways induces expression of IL-25 and IL-17RB in serum and the lungs [43, 44]. In a prior study of human subjects with asthma, $I L 17 R B$ expression in peripheral blood CD4 ${ }^{+} \mathrm{T}$-cells was significantly associated with serum total IgE [45], albeit only in males. Consistent with a key role for IL17RB in atopy in humans, genetic deletion of IL17RB significantly lessens allergic sensitisation in mice [44].

We identified other plausible candidate genes for atopy and atopic asthma. SMPD3, encoding sphingomyelin phosphodiesterase 3 [46], is hypermethylated and downregulated in the small airways of subjects with chronic obstructive pulmonary disease [47]. PIK3R6, an IL-17A gene target in airway smooth muscle cells [48], has been shown to interact with second-hand smoke exposure on forced expiratory volume in $1 \mathrm{~s}$. Other genes, such as ADAMTS7P1, EPN2 and RAB44, have not been previously reported in TWASs and merit further investigation.

DEGs by atopy often overlapped with those for atopic asthma. Most of our top findings were correlated with genotypic data, emphasising a genetic predisposition to atopy. Moreover, a network analysis based on partial correlation revealed close interactions between significant DEGs.

Consistent with known mechanisms of atopy, we found that the top two most enriched pathways were the Th2 and Th1/Th2 activation pathways. Other top pathways in our analysis were that for NGF (a critical factor in the pathogenesis of allergic airway inflammation and proliferation of airway smooth muscle cells) [49] and that for adenine/adenosine salvage I (adenosine has been contraindicated in allergy or severe asthma) [50].

We compared our findings for gene expression and atopic asthma in WBCs with those of a prior meta-analysis of gene expression in airway epithelium and asthma [51]. Of the top 40 DEGs by atopic asthma in our study, seven were differentially expressed by asthma in airway epithelium (supplementary table S6). Some of these differences may be due to inclusion of cases of both atopic and nonatopic asthma in the prior analysis in airway epithelium, while others may indicate true tissue-specific differences in gene expression in atopic asthma. We also compared our results with those of a prior European study of sputum transcriptomics in 104 subjects with moderate-to-severe asthma and 16 healthy volunteers [52]. In that study, three transcriptome-associated clusters were identified, with one of them enriched for the IL-13/Th2 pathway. Of the 20 genes comprising this cluster, seven were also associated with atopy or atopic asthma (FDR-adjusted $\mathrm{p}<0.05$ ) in our meta-analysis (PRSS33, CLC, LGALS12, ALOX15, OLIG2, HRH4 and CCR3).

We mapped the top DEGs in our meta-analysis to the top CpG sites discovered in epigenome-wide association studies (EWASs) of atopy and atopic asthma in blood and airway epithelial samples. None of the genes containing or near the top $30 \mathrm{CpG}$ sites recently associated with atopy in an EWAS of nasal epithelium [53] were DEGs by atopy in our study, but two of the top DEGs by atopic asthma in our study (IL5RA and SIGLEC8) were part of differentially methylated regions by asthma in an EWAS of whole blood [54, 55].

Results from our classification models further support the validity of our top findings, as well as the plausibility of developing predictive transcriptomic panels of atopy and atopic asthma, using WBCs, in future longitudinal studies. Although classification models of atopic asthma using transcriptomic data from airway epithelium in adults have shown greater accuracy (AUC 0.99) [10] than that shown in the current study (AUC 0.84), WBCs are easier to collect than nasal or bronchial epithelium [10].

There were differences in both the type of sample (WBCs versus whole blood) and the platform used to assess gene expression (RNA sequencing versus microarray) between EVA-PR and BAMSE. This, together with differences in ethnicity and sample size between the two studies, likely explains lack of significant replication of our findings in EVA-PR in BAMSE. However, a previous study of the transcriptomics of atopic dermatitis found generally good correlation between the results of RNA sequencing and microarrays, except for genes with low expression levels [56]. Moreover, we found nominal replication at $\mathrm{p}<0.05$, with consistent direction and estimated effect size, for some of our top findings in EVA-PR in BAMSE. Although there were no significant DEGs for atopy or atopic asthma in BAMSE after FDR correction for multiple testing, the top genes associated with atopy or atopic asthma in BAMSE (shown in supplementary table S7) were enriched in pathways for energy-coupled proton and ion transmembrane transport.

In summary, our combined transcriptomic analysis in Puerto Rican and Swedish children identified 59 DEGs by atopy and 40 DEGs by atopic asthma, including SIGLEC8 and IL17RB. Transcriptomic data from WBCs could be used to develop predictive models of atopy and atopic asthma in future prospective studies.

Acknowledgements: We thank all the participating children and families. The BAMSE team would like to thank Charles Auffray (European Institute for Systems Biology and Medicine, Lyon, France) and his team for generating the transcriptomics data. 
Author contributions: W. Chen, E. Melén and J.C. Celedón conceived and designed the study. Y. Jiang, O. Gruzieva and S.K. Merid conducted the primary analysis and interpreted data. E. Forno, T. Wang, E. Acosta-Pérez, G. Canino, I. Kull, J.M. Antó and J. Bousquet participated in data collection and data analysis. Y. Jiang, W. Chen and J.C. Celedón prepared the first draft of the manuscript. All authors reviewed the draft for intellectual content and approved submission of the final version of the manuscript.

Conflict of interest: Y. Jiang has nothing to disclose. O. Gruzieva has nothing to disclose. T. Wang has nothing to disclose. E. Forno has nothing to disclose. N. Boutaoui has nothing to disclose. T. Sun has nothing to disclose. S.K. Merid has nothing to disclose. E. Acosta-Pérez has nothing to disclose. I. Kull has nothing to disclose. G. Canino has nothing to disclose. J.M. Antó has nothing to disclose. J. Bousquet reports personal fees for advisory board work, consultancy and lectures from Chiesi, Cipla, Hikma, Menarini, Mundipharma, Mylan, Novartis, Sanofi-Aventis, Takeda, Teva and Uriach, and owns shares in Kyomed, outside the submitted work. E. Melén has nothing to disclose. W. Chen has nothing to disclose. J.C. Celedón has received research materials from GSK, Merck and Pharmavite, outside the submitted work.

Support statement: This study was supported by grants HL079966, HL117191 and MD011764 (Principal Investigator: J.C. Celedón) from the US National Institutes of Health (NIH). E. Forno's contribution was supported by NIH grant HL125666. Y. Jiang was supported by the China Scholarship Council. The BAMSE cohort was supported by grants from the Swedish Heart-Lung Foundation, the Swedish Research Council, Stockholm County Council (ALF), the Strategic Research Program (SFO) in Epidemiology at Karolinska Institutet and MeDALL (Mechanisms of the Development of ALLergy; European Union grant agreement number 261357). E. Melén is supported by a grant from the European Research Council (grant agreement number 757919, TRIBAL). Funding information for this article has been deposited with the Crossref Funder Registry.

\section{References}

1 Pearce N, Aït-Khaled N, Beasley R, et al. Worldwide trends in the prevalence of asthma symptoms: phase III of the International Study of Asthma and Allergies in Childhood (ISAAC). Thorax 2007; 62: 758-766.

2 Jacobs TS, Forno E, Brehm JM, et al. Underdiagnosis of allergic rhinitis in underserved children. J Allergy Clin Immunol 2014; 134: 737-739.

3 Rosser FJ, Forno E, Cooper PJ, et al. Asthma in Hispanics. An 8-year update. Am J Respir Crit Care Med 2014 189: $1316-1327$.

4 Akinbami LJ, Moorman JE, Bailey C, et al. Trends in asthma prevalence, health care use, and mortality in the United States, 2001-2010. NCHS Data Brief 2012; (94): 1-8.

5 Bjerg A, Sandstrom T, Lundback B, et al. Time trends in asthma and wheeze in Swedish children 1996-2006: prevalence and risk factors by sex. Allergy 2010; 65: 48-55.

6 Demenais F, Margaritte-Jeannin P, Barnes KC, et al. Multiancestry association study identifies new asthma risk loci that colocalize with immune-cell enhancer marks. Nat Genet 2018; 50: 42-53.

7 Waage J, Standl M, Curtin JA, et al. Genome-wide association and HLA fine-mapping studies identify risk loci and genetic pathways underlying allergic rhinitis. Nat Genet 2018; 50: 1072-1080.

8 Yick CY, Zwinderman AH, Kunst PW, et al. Transcriptome sequencing (RNA-Seq) of human endobronchial biopsies: asthma versus controls. Eur Respir J 2013; 42: 662-670.

9 Poole A, Urbanek C, Eng C, et al. Dissecting childhood asthma with nasal transcriptomics distinguishes subphenotypes of disease. J Allergy Clin Immunol 2014; 133: 670-678.

10 Pandey G, Pandey OP, Rogers AJ, et al. A nasal brush-based classifier of asthma identified by machine learning analysis of nasal RNA sequence data. Sci Rep 2018; 8: 8826.

11 Pascual M, Roa S, Garcia-Sanchez A, et al. Genome-wide expression profiling of B lymphocytes reveals IL4R increase in allergic asthma. J Allergy Clin Immunol 2014; 134: 972-975.

12 Howrylak JA, Moll M, Weiss ST, et al. Gene expression profiling of asthma phenotypes demonstrates molecular signatures of atopy and asthma control. J Allergy Clin Immunol 2016; 137: 1390-1397.

13 Croteau-Chonka DC, Qiu W, Martinez FD, et al. Gene expression profiling in blood provides reproducible molecular insights into asthma control. Am J Respir Crit Care Med 2017; 195: 179-188.

14 Bird HR, Davies M, Duarte CS, et al. A study of disruptive behavior disorders in Puerto Rican youth: II. Baseline prevalence, comorbidity, and correlates in two sites. J Am Acad Child Adolesc Psychiatry 2006; 45: 1042-1053.

15 Brehm JM, Acosta-Perez E, Klei L, et al. Vitamin D insufficiency and severe asthma exacerbations in Puerto Rican children. Am J Respir Crit Care Med 2012; 186: 140-146.

16 Forno E, Cloutier MM, Datta S, et al. Mouse allergen, lung function, and atopy in Puerto Rican children. PLoS One 2012; 7: e40383.

17 Andrews S. FastQC: a quality control tool for high throughput sequence data. 2010. www.bioinformatics. babraham.ac.uk/projects/fastqc Date last accessed: March 8, 2019.

18 Krueger F. Trim Galore!: a wrapper tool around Cutadapt and FastQC to consistently apply quality and adapter trimming to FastQ files. 0.4. 2015. www.bioinformatics.babraham.ac.uk/projects/trim_galore Date last accessed: March 8, 2019.

19 Martin M. Cutadapt removes adapter sequences from high-throughput sequencing reads. EMBnet J 2011; 17: $10-12$.

20 Dobin A, Davis CA, Schlesinger F, et al. STAR: ultrafast universal RNA-seq aligner. Bioinformatics 2013; 29: $15-21$.

21 Li B, Dewey CN. RSEM: accurate transcript quantification from RNA-Seq data with or without a reference genome. BMC Bioinformatics 2011; 12: 323.

22 Brehm JM, Acosta-Pérez E, Klei L, et al. African ancestry and lung function in Puerto Rican children. J Allergy Clin Immunol 2012; 129: 1484-1490.

23 Das S, Forer L, Schönherr S, et al. Next-generation genotype imputation service and methods. Nat Genet 2016; 48: 1284-1287.

24 McCarthy S, Das S, Kretzschmar W, et al. A reference panel of 64,976 haplotypes for genotype imputation. Nat Genet 2016; 48: 1279-1283. 
25 Ballardini N, Bergström A, Wahlgren C-F, et al. IgE antibodies in relation to prevalence and multimorbidity of eczema, asthma, and rhinitis from birth to adolescence. Allergy 2016; 71: 342-349.

26 Hallberg J, Ballardini N, Almqvist C, et al. Impact of IgE sensitization and rhinitis on inflammatory biomarkers and lung function in adolescents with and without asthma. Pediatr Allergy Immunol 2019; 30: 74-80.

27 Love MI, Huber W, Anders S. Moderated estimation of fold change and dispersion for RNA-seq data with DESeq2. Genome Biol 2014; 15: 550.

28 Lee $\mathrm{CH}$, Cook S, Lee JS, et al. Comparison of two meta-analysis methods: inverse-variance-weighted average and weighted sum of z-scores. Genomics Inform 2016; 14: 173-180.

29 Schwarzer G. meta: an R package for meta-analysis. R News 2007; 7: 40-45.

30 Shabalin AA. Matrix eQTL: ultra fast eQTL analysis via large matrix operations. Bioinformatics 2012; 28 : 1353-1358.

31 Krämer A, Green J, Pollard J Jr, et al. Causal analysis approaches in Ingenuity Pathway Analysis. Bioinformatics 2013; 30: 523-530.

32 Wang T, Ren Z, Ding Y, et al. FastGGM: an efficient algorithm for the inference of Gaussian graphical model in biological networks. PLoS Comput Biol 2016; 12: e1004755.

33 Lonsdale J, Thomas J, Salvatore M, et al. The Genotype-Tissue Expression (GTEx) project. Nat Genet 2013; 45: 580-585.

34 Newman AM, Liu CL, Green MR, et al. Robust enumeration of cell subsets from tissue expression profiles. Nat Methods 2015; 12: 453-457.

35 Yokoi $\mathrm{H}$, Choi $\mathrm{OH}$, Hubbard W, et al. Inhibition of FceRI-dependent mediator release and calcium flux from human mast cells by sialic acid-binding immunoglobulin-like lectin 8 engagement. J Allergy Clin Immunol 2008; 121: 499-505.

36 Choy DF, Jia G, Abbas AR, et al. Peripheral blood gene expression predicts clinical benefit from anti-IL-13 in asthma. J Allergy Clin Immunol 2016; 138: 1230-1233.

37 Gao P-S, Shimizu K, Grant AV, et al. Polymorphisms in the sialic acid-binding immunoglobulin-like lectin-8 (Siglec-8) gene are associated with susceptibility to asthma. Eur J Hum Genet 2010; 18: 713-719.

38 Hwang SM, Uhm TG, Lee SK, et al. Olig2 is expressed late in human eosinophil development and controls Siglec-8 expression. J Leukoc Biol 2016; 100: 711-723.

39 Gomez G, Nardone V, Lotfi-Emran S, et al. Intracellular adenosine inhibits IgE-dependent degranulation of human skin mast cells. J Clin Immunol 2013; 33: 1349-1359.

40 Forno E, Wang T, Yan Q, et al. A multiomics approach to identify genes associated with childhood asthma risk and morbidity. Am J Respir Cell Mol Biol 2017; 57: 439-447.

41 Vercelli D. Discovering susceptibility genes for asthma and allergy. Nat Rev Immunol 2008; 8: 169-182.

42 Chen W, Wang T, Pino-Yanes $\mathrm{M}$, et al. An epigenome-wide association study of total serum IgE in Hispanic children. J Allergy Clin Immunol 2017; 140: 571-577.

43 Weathington NM, Kanth SM, Gong Q, et al. IL-4 induces $I L 17 R b$ gene transcription in monocytic cells with coordinate autocrine IL-25 signaling. Am J Respir Cell Mol Biol 2017; 57: 346-354.

44 Petersen BC, Budelsky AL, Baptist AP, et al. Interleukin-25 induces type 2 cytokine production in a steroid-resistant interleukin-17RB ${ }^{+}$myeloid population that exacerbates asthmatic pathology. Nat Med 2012; 18: 751-758.

45 Hunninghake GM, Chu JH, Sharma SS, et al. The CD4 ${ }^{+}$T-cell transcriptome and serum IgE in asthma: IL17RB and the role of sex. BMC Pulm Med 2011; 11: 17

46 Filosto S, Castillo S, Danielson A, et al. Neutral sphingomyelinase 2: a novel target in cigarette smoke-induced apoptosis and lung injury. Am J Respir Cell Mol Biol 2011; 44: 350-360.

47 Vucic EA, Chari R, Thu KL, et al. DNA methylation is globally disrupted and associated with expression changes in chronic obstructive pulmonary disease small airways. Am J Respir Cell Mol Biol 2014; 50: 912-922.

48 Dragon S, Hirst SJ, Lee TH, et al. IL-17A mediates a selective gene expression profile in asthmatic human airway smooth muscle cells. Am J Respir Cell Mol Biol 2014; 50: 1053-1063.

49 Yang Y-G, Tian W-M, Zhang H, et al. Nerve growth factor exacerbates allergic lung inflammation and airway remodeling in a rat model of chronic asthma. Exp Ther Med 2013; 6: 1251-1258.

50 Layland J, Carrick D, Lee M, et al. Adenosine: physiology, pharmacology, and clinical applications. JACC Cardiovasc Interv 2014; 7: 581-591.

51 Tsai Y-H, Parker JS, Yang IV, et al. Meta-analysis of airway epithelia gene expression in asthma. Eur Respir J 2018; 51: 1701962.

52 Kuo C-HS, Pavlidis S, Loza M, et al. T-helper cell type 2 (Th2) and non-Th2 molecular phenotypes of asthma using sputum transcriptomics in U-BIOPRED. Eur Respir J 2017; 49: 1602135.

53 Forno E, Wang T, Qi C, et al. DNA methylation in nasal epithelium, atopy, and atopic asthma in children: a genome-wide study. Lancet Respir Med 2019; 7: 336-346.

54 Reese SE, Xu CJ, den Dekker HT, et al. Epigenome-wide meta-analysis of DNA methylation and childhood asthma. J Allergy Clin Immunol 2018; in press [https://doi.org/10.1016/j.jaci.2018.11.043].

$55 \mathrm{Xu}$ CJ, Soderhall C, Bustamante M, et al. DNA methylation in childhood asthma: an epigenome-wide meta-analysis. Lancet Respir Med 2018; 6: 379-388.

56 Suárez-Fariñas M, Ungar B, da Rosa JC, et al. RNA sequencing atopic dermatitis transcriptome profiling provides insights into novel disease mechanisms with potential therapeutic implications. J Allergy Clin Immunol 2015; 135 $1218-1227$. 